Copyright (C) 2013 IEEE. Personal use of this material is permitted. Permission from IEEE must be obtained for all other uses, in any current or future media, including

reprinting/republishing this material for advertising or promotional purposes, creating new collective works, for resale or redistribution to servers or lists, or reuse of any copyrighted component of this work in other works. 


\title{
Health Service Discovery and Composition in Ambient Assisted Living: the Australian Type 2 Diabetes Case Study
}

\author{
Davor Meersman \\ Curtin Business School \\ Fedja Hadzic \\ Jeff Hughes \\ Department of Computing School of Pharmacy \\ Curtin University \\ Perth, Australia \\ \{d.meersman, f.hadzic, j.d.hughes\}@curtin.edu.au
}

\author{
Ivan Razo-Zapata Pieter De Leenheer \\ Business, Web and Media \\ VU University Amsterdam \\ Amsterdam, The Netherlands \\ \{i.s.razozapata, \\ p.g.m.de.leenheer\}@vu.nl
}

\begin{abstract}
This paper is situated in the Ambient Assisted Living (AAL) services domain and offers a twofold contribution to the state of the art. We contribute to the health informatics domain by applying a service value network (SVN) approach to automatically match medical practice recommendations based on patient sensor data in a home care monitoring context to health services provided by a network of service providers. We contribute to the area of SVN composition by replacing current assumptions that customers are actively involved in explicating their service requirements with a more tacit approach where requirements are derived from patterns in sensor readings (and corollary diagnosis) based on validated rules on the customer side as well as on the supplier side. We demonstrate our contributions with an SVN composition based on an initial set of 493 patient profiles in the context of Type 2 Diabetes management in Australia.
\end{abstract}

\section{Introduction}

Ambient Assisted Living (AAL) has been identified as a viable option to mitigate the impact of costs associated with ageing populations in industrialized societies [1]. AAL is a relatively young domain that faces a number of technological challenges, i.a. heterogeneity, domain knowledge formalization, and integration [2]. Heterogeneity in AAL refers to the nature of standalone, closed systems provided by different suppliers with diverging knowledge and technologies. Challenges associated with domain knowledge formalization have to do with the difficulty of formalizing heath domain knowledge for machine processing. Finally, integration challenges are related to the above heterogeneity issues and disparate data sources.

The contribution of this paper is twofold: (i) We contribute to the health informatics domain by applying a service value network (SVN) approach to automatically match patient sensor data in a home care monitoring context to health services provided by a network of service providers. The process is based on machine-readable rules extracted from national medical guidelines, and health service networks that are inferred from medical benefits schedule item listings. In essence we cover the full spectrum from sensor reading to service delivery using already available data, although currently not all relevant data is used in calculating service offers in our framework. The contributions are relevant for both researchers and practitioners in the health (informatics) domain.

(ii) We contribute to the area of SVN composition by replacing current assumptions that customers are actively involved in explicating their service requirements with a more tacit approach where requirements are derived from patterns in sensor readings (and corollary diagnosis) based on validated rules on the customer side as well as the supplier side.

We demonstrate our contributions with an SVN composition based on an initial set 493 patient profiles in the context of Type 2 Diabetes management. We have extracted rules for diabetes diagnosis and management from the Australian National Health and Medical Research Council (NHMRC) [7] medical guidelines. For the service composition, we rely on the listed medical services of the Medicare Benefits Schedule (MBS) [8] of the Australian government. The framework including all formalizations, rules, itemizations, mappings, and resulting service compositions have been validated by medical domain experts.

This paper is organized as follows: Section 2 discusses the background components and motivations. Section 3 outlines the approach. Section 4 describes the experiments and the case study, followed by a discussion in Section 5 and concluding with Section 6. 


\section{Background and motivation}

In this section we outline the main areas, components, and motivations of our approach. These are Ambient Assisted Living, the NHMRC medical practice recommendations, the MBS service listings, and Service Value Networks.

\subsection{Ambient Assisted Living}

The population of industrialized nations is undergoing significant demographic changes that have implications on the nature of health services in the future. In Europe, the population older than 65 is projected to be more than $30 \%$ by 2060 [3]. In Australia, we see similar projections with an increase of people over 65 to $23 \%$ by 2056 [4].

The associated cost of the related increase in care needs is expected to place a significant burden on economies [2]. This effect is further exacerbated by the old age dependency ratio (i.e. the population older than 65 divided by the working age population supporting them), which is expected to rise from $25 \%$ to $53 \%$ by 2060 in Europe [3], and from $20 \%$ to $38 \%$ by 2056 in Australia [4]. This means that for every old person there will be two or three people of working age that can support that person, compared to four or five people now. In Australia, the situation is further encumbered by the fact that a significant part of the population lives and will continue to live in remote and rural areas with both lower and more expensive access to traditional healthcare services [5].

Assisted living solutions underpinned by ambient intelligence technology have been identified as a viable option to mitigate the impact of the associated cost of the demographic changes faced by industrialized nations [1]. Ambient Assisted Living (AAL) technologies can help provide autonomy to elderly and disabled people, allow them to live at home individually for longer, and increase their quality of life, whilst at the same time relieving some of the economic burden on public health care systems.

Systems focusing on supporting people with special needs in their home environment are called Home Care Systems (HCS). Technologies underpinning home care have various labels. 'Assisted Living' refers to devices and services that help people stay at home longer autonomously. 'Assistive Technologies' refer to devices that aid with daily living of patients. 'Telehealth' and 'Telecare' refer to remotely monitoring and supporting patients. 'Smart Home" refers to home automation and monitoring via sensor networks [6]. In our case of Type 2 Diabetes management at home, two types of sensors can be used to remotely monitor the health of patients: blood pressure sensors for monitoring hypertension, and blood glucose sensors (which can be part of continuous blood glucose monitoring systems) for monitoring glycemic control. Lipids are measured as well for hyperlipidaemia in the context of diabetes, but can currently not be implemented via remote sensors.

As a domain, AAL is relatively young and in the early stages of its development. Kleinberger et al. [2] define a number of technological challenges the AAL domain is currently facing: (1) Adaptivity: systems need to monitor their environment and adapt themselves constantly. (2) Natural interactions: systems need to provide interfaces for users with varying needs. (3) Heterogeneity: systems are closed, standalone, and provided by different suppliers with diverging knowledge and technologies. (4) Domain knowledge formalization: domain knowledge that is difficult to formalize needs to be transformed for processing. (5) Elderly stakeholders: the main stakeholders of AAL have generally low degrees of computer literacy and variable degrees of mental clarity, alertness and memory function, creating interface constraints. (6) Low acceptance: systems that are marketed as solely assisting with health problems have low acceptance rates because of the social stigma associated with them. (7) Integration of available technologies: AAL systems and services are characterized by heterogeneity and disparate data sources, which offers integration challenges. (8) Immaturity: although it is generally expected that AAL will be a huge market, there is only limited knowledge about what the products will look like, what their economic viability will be, who will provide them, how they will integrate, etc.

The initial focus of our proposed framework is on tackling challenges 3, 4 and 7. This will form the basis for tackling the remaining challenges in the domain. Our proposed framework aims to integrate the 'medical practice' side of healthcare with the 'business' side of it through the application of a service value network approach (AAL challenge 7). The medical practice side is embodied by the medical practice guidelines of the NHMRC guidelines [7]. The business side is represented by medical service listings in the Medicare Benefits Schedule [8]. Each provider claims for the services they perform, therefore, a service network can be derived from the claims providers make. In a sense, this novel approach using existing medical data can be seen as 'opening the playing field' for service providers (AAL challenge 3 ), as their actions in order to participate in the business ecosystem need not go beyond what they are doing already anyway. Initial and reusable mappings by domain experts between medical practice recommendations (or 'functional 
consequences') and medical service listings create a very straightforward integration in the domain.

In order to create the degree of automation desired in an AAL context, rules were extracted from the complex and generally difficult to formalize NHMRC guidelines (AAL challenge 4). The content was further itemized to a state where the rules and content become useful as a form of 'sensor-driven, automated healthcare'.

\subsection{National Health and Medical Research Council}

The National Health and Medical Research Council (NHMRC) in Australia is a government body promoting the development and maintenance of public and individual health standards. It serves the functions of research funding and development of advice and draws upon the resources of all components of the national health system (including governments, medical practitioners, nurses and allied health professionals, researchers, teaching and research institutions, public and private program managers, service administrators, community health organisations, social health researchers and consumers) [7].

The NHMRC guidelines are developed by a team of health and medical field specialists, with the purpose of providing a general guide to appropriate practice. They are based on the best evidence at the time of development and provide a set of principles and recommendations to assist in decision-making. These are expected to be followed by the general practitioner/clinician, subject to their judgement and patient's preference in each individual case.

\subsection{Medicare Benefits Schedule}

The Medicare Program provides access to medical and hospital service for Australian residents. Medicare benefits or 'rebates' are expressed as a percentage of the total cost of a medical service, ranging from $75 \%$ to $100 \%$. Benefits are claimed by service providers and patients and are reimbursed by the Australian Government [8].

The Medicare Benefits Schedule (MBS) is a comprehensive listing of all professional medical services reimbursed through the Medicare Program. Each health service has a unique ID number, a name, a description and the fee/benefit of that service in Australian dollars. The MBS can be consulted in document form, via a web interface, and in a downloadable xml format. Services are related to each other by explanations containing hyperlinks. The descriptions of services also contain business rules, i.e. constraints on the use of the services from both the patient and provider perspective (e.g. patient eligibility, service history, provider eligibility, cost constraints, etc.).

\subsection{Service Value Networks}

As opposed to value chains, which are sequences of activities each adding a part of the value to a production process, in SVNs value is co-created in a decentralized setting. A service value network is a flexible and dynamic web of enterprises and final customers who reciprocally establish relationships with each other for delivering an added-value service to a final customer (see Razo-Zapata et al. [9]; Hamilton [10]; Allee [11]; and Lovelock and Wirtz [12]. The key challenge of SVN composition is to automatically match and bundle relevant competences in a network such that they deliver the value (i.e. a service) required by a customer. SVNs are usually organized in different tiers: at B2C side, service providers aim to offer relevant competences in fulfillment of a customer need; at B2B side, service enablers support the technology space that can make these services possible [13]; hence e-services.

e3value $[14,15]$ is a well-known SVN composition approach. It features ontologies to analyze and model perspectives of customers and providers on service needs. Inspired by service marketing and management theory, the conceptualization of services focuses on value aspects, rather than merely computertechnical aspects as found in most service-oriented computing paradigms.

\section{Approach}

In this section we describe our approach in terms of SVN composition. We start off with a general overview of the methodology and describe our modifications to the existing e3value framework for the healthcare domain and case study. We then elaborate on the methodology used for converting sensor data into rule-based and validated functional consequences that can be used in SVN composition. We then continue to explain the process used to map healthcare services and service providers to these functional consequences, again with the purpose of SVN composition.

\subsection{Overview}




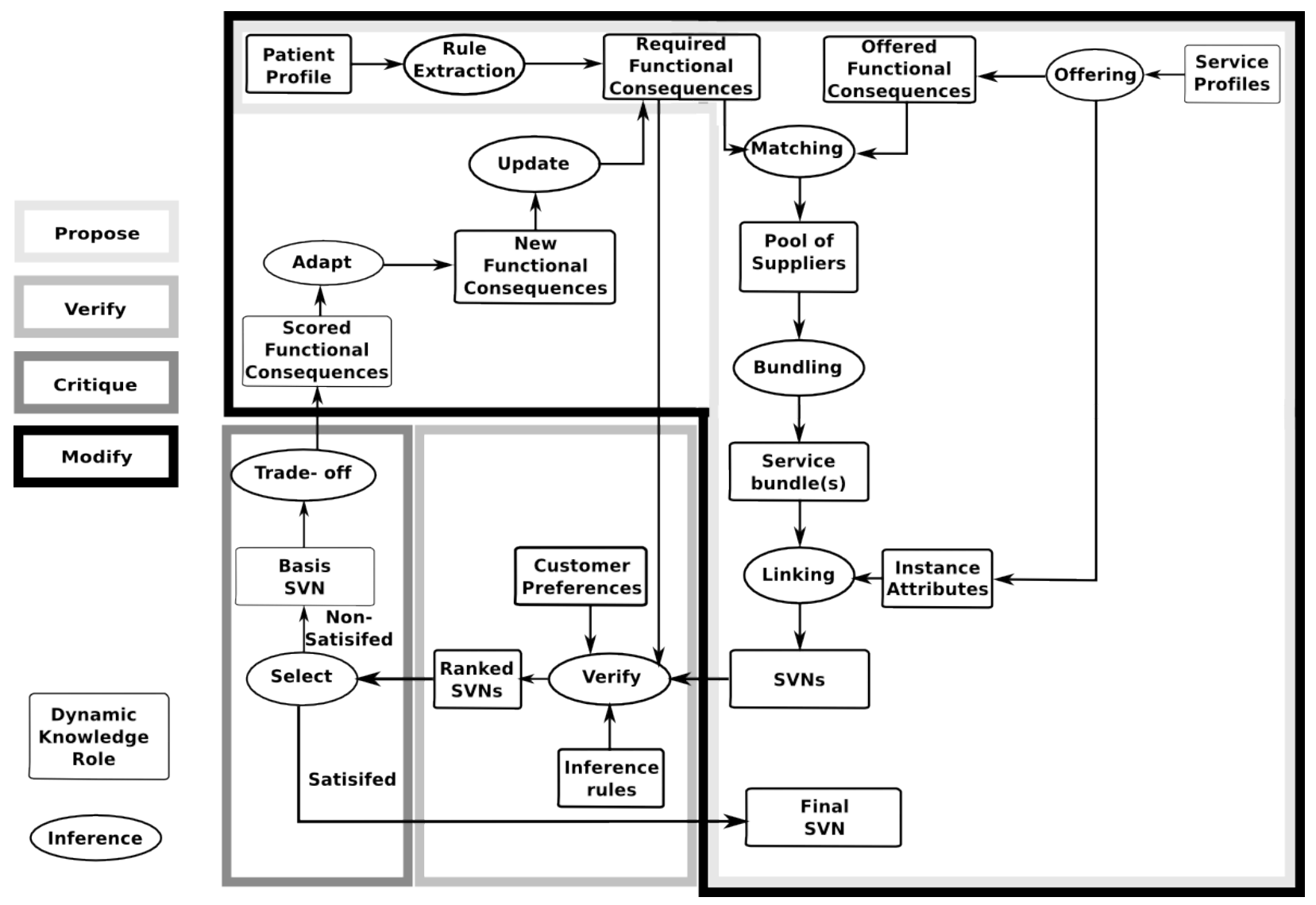

Fig. 1 Service Value Network Composition

The purpose of a SVN is to find an optimal service composition (supplier perspective) for a given customer need (customer perspective). In e3value, this is done via a method described in $[16,17,18]$. The method consists of four subtasks as depicted in Fig. 1: Propose, Verify, Critique, and Modify [19]. Discussing the entire method is not the essence of this paper, which is mainly focused on the Propose aspect. We will however give a brief overview of the other aspects as well.

(1) The Propose subtask is concerned with proposing an appropriate solution for a need. It involves a number of subprocesses. (a) Laddering is a marketing practice where marketers explore how customers link specific product properties to higherlevel values. In SVNs, the process is used to transform customer needs into what is termed 'functional consequences' (FCs). FCs are actionable items that can be satisfied by suppliers. Traditionally, FCs are derived from explicit customer needs. However, in many contexts, and especially those where end users have no clear idea, or insufficient knowledge, it is prohibitively difficult or unadvisable to have users explicate their needs [20]. Therefore, in our approach we replace the laddering process with a more tacit approach involving an extracted rule base that acts on sensor readings and recommends FCs according to the rule base (see section 3.2: 'Rule extraction and mapping'). Although the end user is still the recipient of the service, the translation of their needs to FCs is done outside of their grasp, i.e. in an ambient manner. (b) Offering is a similar process to laddering, but then from the supplier perspective. In this process, suppliers translate their offers (assets and competencies) to FCs as well. In our approach, we substitute this traditional aspect with mappings between services and FCs that have been validated by health domain experts (see section 3.3: 'Service characteristics and mappings'). The reasoning behind this is that in a context of health services, a validation of the mappings between services and FCs is called for because of accountability and accuracy considerations, i.e. we need to make sure that the right service is delivered according to agreed-upon medical practice recommendations. (c) Matching concerns the matching of customer with supplier in terms of FCs, additionally constrained by medical practice considerations and customer preference on the customer side, and business rules on the supplier side. 
In our case study, the matching process is appended in favor of a direct mapping based on CustomerRules, ProviderRules and ServiceRules. The matching process results in a pool of service providers. (d) Bundling is the process where meaningful combinations of services and service providers are made to satisfy the customer need. The output of this process is a set of service bundles that describe $\mathrm{B} 2 \mathrm{C}$ relationships in terms of value exchanges. Finally, in the (5) Linking stage we solve any B2B dependencies that might exist between various service providers.

The following subtasks are not at the core of this paper, but will be discussed briefly with the aim of being complete.

(2) The Verify subtask refers to the process of checking whether the SVN designs satisfy functional requirements. Once the SVNs have been composed, the verification subtask determines (a) whether the SVN offers the required FCs and (b) how it fits the customer requirements.

(3) The Critique subtask identifies the source of failure in case of unsuccessful design and allows for the designs to be improved before deployment.

(4) Finally, the Modify subtask takes input from monitoring the SVN performance (customer feedback, patient health status, etc.) and allows for improvements after deployment.

We will now discuss our approach in transforming the necessary data from customer and service provider sides to FCs.

\subsection{Rule extraction and mapping}

For the rule extraction, we have focused on the utilization of NHMRC guidelines related to treatment of patients diagnosed with Type 2 Diabetes. The guidelines provide detailed pharmacological and nonpharmacological advice on how to manage the patient's hypertension, hyperlipidaemia and hyperglycaemia in the light of their existing biological parameters and clinical data. While the guidelines are very extensive in detailing the evidence for stated recommendations, including previous literature, academic research findings, health practice case studies, etc., we have focused on the summary of recommendations within the guidelines. This is where the majority of confirmed consistently applicable recommendations for a typical patient profile are found.

We have formalized the main NHMRC guidelines and recommendations related to hypertension (blood pressure control), hyperlipidaemia (lipid control) and hyperglycaemia (blood glucose control) in a format that could be implemented within the proposed framework. This formalization was in form of IFTHEN rules where the precedent of a rule indicates the set of preconditions that must hold, while the consequent indicates the treatment recommendation. These rules were verified and validated by domain experts. In consultation with the domain experts the rules were further enriched to reflect a particular stage of the treatment and when more specific information was available it was added to the more general rules extracted (e.g. specific medicine used, and which medicine should/should not be used in combination with other medicine/treatment). This resulted in a total of 131 rules out of which 90 were related to blood pressure control, 21 to blood glucose control and 20 to lipid control. Note that if the characteristics of a patient satisfy the pre-conditions of multiple rules, then multiple recommendations for treatment would be provided within the guidelines.

Note that henceforth we will use the general term attribute to refer to a set of measurements (e.g. blood pressure, glucose level etc.) or patient characteristics. In the formulization that follows, for simplicity purposes we assume that the set of conditions/measurement values of attributes from NHMRC guidelines and observed from patients are represented by either a true or false value for a particular attribute.

Let the set $A=\left\{a_{1}, a_{2}, \ldots, a_{|A|}\right\}$ denote the attributes, $A C$ the set of true/false conditions on $a_{i}(i=$ $1, \ldots,|A|)$, and $a c_{i} p$ be the true/false value of attribute $a_{i}$ for a patient $p$.

The set of treatment recommendations is denoted as $T=\left\{t_{1}, t_{2}, \ldots, t_{|\mathrm{T}|}\right\}$ and the rule set is denoted as $R=$ $\left\{r_{1}, r_{2}, \ldots, r_{|R|}\right\}$.

Each rule $r_{n}(n=(1, \ldots,|R|)$ is an implication of the form of $x \rightarrow y$ where $x \subseteq A C$ and $y \in T$.

The dataset containing patient observations/profiles is denoted as $O=\left\{O_{1}, O_{2}, \ldots\right.$, $\left.O_{|\mathrm{O}|}\right\}$, where $O_{p}=\left\{a c_{1} p, a c_{2} p, \ldots, a c_{|A|} p\right\}$ denotes the set of observations of a particular patient $p(p=(1, \ldots$, $|O|)$.

Given $O_{p}$ and $R$, the system will form a set of treatment recommendations $T p$ for a patient $p$ as follows: $\forall t_{i} \in T$, if $\exists r_{n}=x \rightarrow y$ where $x \subseteq O_{p}$ and $y=t_{i}$ then $T_{p}=T_{p} \cup t_{i}$.

Note that recommendations are not unique for each rule, as different patient profile/observations may provoke the same treatment recommendation. Each treatment recommendation $t_{i} \in T$ was assigned a unique $\mathrm{FC}$ identifier $\left(f_{c}\right)$.

\subsection{Service characteristics and mapping}

In order to bootstrap the services, a broad selection of diabetes related services was extracted from the 
Medicare Benefits Schedule (MBS) database. This list was narrowed down by domain experts to include any and all services relating to Diabetes Type 2. This process amounted to a list of 32 services. The set of services will be denoted as $S=\left\{s_{1}, s_{2}, \ldots, s_{32}\right\}$ and each service has nine characteristics associated with it, denoted as $s_{i} c_{j}(i=(1, \ldots, 32) ; j=(1, \ldots, 9))$.

The service characteristics that were relevant in the selection and composition of diabetes-related services using our framework were extracted from the MBS listings. These characteristics are formalised as follows:

(1) ServiceID: a unique identifier provided by Medicare. The ID number contains information about the 'family' of services a particular service belongs to. The first and second digits typically identify category and subcategory, and the last digit uniquely identifies the service. For example $s_{1} c_{1}=701, s_{5} c_{1}=2517, s_{6} c_{1}=$ 2518, $s_{7} c_{1}=2521, s_{8} c_{1}=2522$ and $s_{26} c_{1}=6651$, all belong to the same 'family' (i.e. Consultations performed by General Practitioners in Vocational Register (REG-GP) and/or Fellow of the Royal Australian College of General Practitioners (FRACGP)). They differ in terms of length (i.e. time needed to execute the service) and/or place (i.e. place of execution, usually defined as in or outside a facility).

(2) Description: a short description for human interpretation, e.g. $s_{1} c_{2}=$ "Brief Health Assessment", $s_{5} c_{2}=$ 'Consultation at consulting rooms' or $s_{26} c_{2}=$ 'Quantitation of glycosylated haemoglobin'.

(3) Duration: refers to the time needed to execute a service. Duration is expressed in a minute interval, e.g. $s_{5} c_{3}=0-20 ; s_{7} c_{3}=20-40 ; s_{9} c_{3}>40$.

(4) Fee: refers to the total amount in Australian dollars that will be charged for the service, e.g. $s_{5} c_{4}=$ $\$ 35.6 ; s_{26} c_{4}=\$ 16.9$.

(5) Benefit \%: refers to the percentage of the fee that can be claimed for reimbursement, e.g. $s_{5} c_{5}=$ $100 \% ; s_{26} c_{5}=75 \%$.

(6) Benefit: reflects the actual amount that can be claimed for reimbursement, e.g. $s_{5} c_{6}=\$ 35.6 ; s_{26} c_{6}=$ $\$ 12.7$.

(7) CustomerRule: pertains to constraints for service delivery related to the status of the customer, e.g. $s_{1} c_{7}=$ " $I F$ diabetes established THEN use $s_{5}$ ", $s_{8} c_{7}=$ "IF number of patients $>7$ THEN $s_{8} c_{4}=s_{7} c_{4} *$ (number of patients * 1.9))".

(8) ProviderRule: pertains to constraints for services of which the delivery can only be performed by service providers with a certain status, e.g. $s_{5} c_{8}=$ "IF provider is REG-GP or FRACGP THEN valid ELSE use $s_{11}$ ".

(9) ServiceRule: refers to rules regarding the relation of a service to other services, e.g. service items that are iterations of each other; that are identical services with a different length, service dependencies, etc. For example, $s_{28} c_{9}=$ "Valid if patient has been delivered $s_{27}$ " (in this case $s_{27}$ is an assessment to be allowed to group service $s_{28}$ ).

Once all services were itemized, they were mapped to the FCs under guidance of a domain expert. Mappings were non-exclusive, i.e. an FC can be satisfied with multiple services and a single service can satisfy multiple FCs. Hence, each FC was mapped to one or more services that it requires to produce the set of mappings $M=\left\{m_{1}, m_{2}, \ldots, m_{|M|}\right\}$, where each $m \in$ $M$ is a 2-tuple denoted as $\left(f c_{i}, m s\right)$, where $f c_{i}(i=(1, \ldots$, $|T|)$ is a FC identifier and $m s \subseteq S$.

Identical services of varying duration were assigned exclusive FCs within their family. For example, $f_{c_{2}}$ ("Measure blood pressure") can be satisfied by the services $s_{5}\left(s_{5} c_{3}=0-20\right) ; s_{7}\left(s_{7} c_{3}=20\right.$ $40)$; and $s_{9}\left(s_{9} c_{3}=>40\right)$. A blood pressure measurement only takes short time, and if that is all that is needed it is performed by $s_{5}$. However, if patient requires one or more additional FCs at the same time, $f c_{2}$ would be amalgamated into the service with longer duration (e.g. $\left.s_{9}\right)$.

\section{Case study and evaluation}

\subsection{Experimental setup}

Customer perspective: we ran 493 different patient profiles (i.e. $|O|=493$ ) through the rule base with 111 rules (i.e. $|R|=111$ ). Note that a single profile can trigger multiple rules resulting in multiple treatment recommendations or FC. From the 493 profiles, 1090 rules were triggered while 64 profiles did not trigger any rules (no treatment was required). Each FC was mapped to one or more services, i.e. the mapping $M$ $(|M|=27)$ was produced between $27 \mathrm{FCs}\left(f_{c_{1}}, \ldots, f_{c_{27}}\right)$ (shown on the bottom left of Fig. 2) and the 32 services ( $S=\left\{s_{1}, \ldots, s_{32}\right\}$ ) (shown on the bottom right of Fig. 2). Although all profiles were different, the requested FCs were often very similar (as can be expected in a context of standardized medical treatment). For this reason, we opted to select a sample of 5 very different profiles for the SVN composition for demonstration purposes.

Service provider perspective: we used 10 different service providers (denoted as $S P_{1}, \ldots, S P_{10}$ ) who between them had 45 single services (i.e. unique service-provider combinations). Each $S P$ can provide multiple services as can be seen in the top right table of Fig. 2 (where we show all services each $S P$ provides). The total pool of service providers could satisfy all possible FCs. 


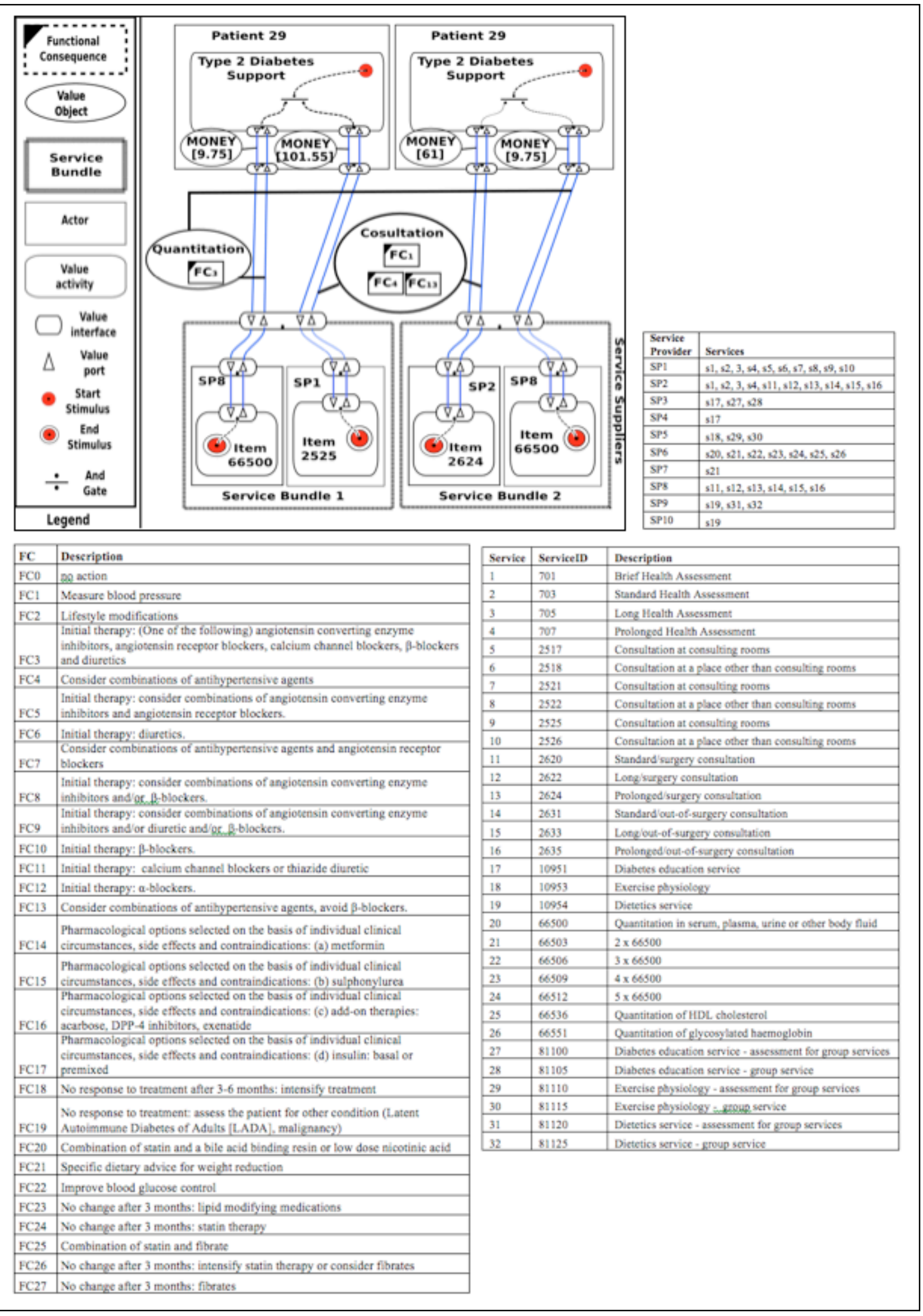

Fig. 2 SVN, FCs, Services and Service Providers 
Service value networks: The 5 profiles generated up to 20 alternative SVNs each. Although it is possible to generate more, the number was restricted for practical purposes. Out of our sample of 493 patient profiles, we selected a patient profile $p=29$ for discussion purposes as this profile triggered many rules and had an interesting, varied SVN composition as a result. In the following section, we will elaborate on the process using this patient profile as an example.

\subsection{Case study: Patient Number 29}

Patient Number 29 has established Type 2 diabetes and is already part of a cycle of care involving his condition. He has had prior advice to make lifestyle modifications, and has a heart condition. His blood pressure sensor indicates that his blood pressure has risen above a predefined threshold, which has triggered a number of rules, resulting in a set of required FCs:

\section{Recommendations/FCs for the patient: Matched to Rule 4 \\ IF Date BP measured $>6$ months $=$ yes THEN measure blood pressure (maps to $f c_{1}$ )}

Matched to Rule 8

IF One of the following applied? Angiotensin converting enzyme inhibitors, angiotensin receptor blockers| calcium channel blockers, beta-blockers and diuretics $=$ no $A N D$

$\mathrm{BP}<125 / 75 \mathrm{mmHg}=$ no $A N D$

Proteinuria $>1 \mathrm{~g} /$ day=yes $A N D$

Combinations of antihypertensive agents $=$ no $A N D$ DiscussedLifeModificationFactors $=$ yes THEN High blood pressure (it should be $<125 / 75$ $\mathrm{mmHg}$ ): Initial therapy: (One of the following) angiotensin converting enzyme inhibitors| angiotensin receptor blockers, calcium channel blockers, beta-blockers and diuretics (maps to $f c_{3}$ )

Matched to Rule 29

IF $\mathrm{BP}<125 / 75 \mathrm{mmHg}=$ no

Heart Failure $=$ yes $A N D$

Proteinuria $>1 \mathrm{~g} /$ day=yes $A N D$

Combinations of antihypertensive agents $=$ no $A N D$

DiscussedLifeModificationFactors $=$ yes $A N D$

Combinations of angiotensin converting enzyme

inhibitors and/or diuretic and/or beta-

blockers=yes $T H E N$ high blood pressure (it should be $<125 / 75 \mathrm{mmHg}$ ): Combinations of

antihypertensive agents (maps to $\mathrm{f}_{4}$ )

Matched to Rule 44

IF $\mathrm{BP}<125 / 75 \mathrm{mmHg}=$ no $A N D$

Proteinuria $>1 \mathrm{~g} /$ day=yes $A N D$
Combinations of antihypertensive agents $=$ no $A N D$

DiscussedLifeModificationFactors=yes $A N D$

Intermittent Claudication=yes $T H E N$ high blood pressure (it should be $<125 / 75 \mathrm{mmHg}$ ): Consider combinations of antihypertensive agents, avoid beta-blockers. (maps to $f c_{13}$ )

The rules have triggered a treatment recommendation $T_{29}=\left\{c_{1}, f c_{3}, f c_{4}, f c_{13}\right\}$ for patient $p=$ 29. This serves as the input for the SVN composition process.

\section{SVN composition:}

The set of recommendations $T_{29}=\left\{f_{1}, f c_{3}, f c_{4}\right.$, $\left.f c_{13}\right\}$ are satisfied by services $s_{5}\left(s_{5} c_{1}=2517\right), s_{7}$ $\left(s_{7} c_{1}=2521\right), \quad s_{9} \quad\left(s_{9} c_{1}=2525\right), \quad s_{11} \quad\left(s_{11} c_{1}=2620\right), \quad s_{12}$ $\left(s_{12} c_{1}=2622\right), s_{13}\left(s_{13} c_{1}=2624\right), s_{20}\left(s_{20} c_{1}=66500\right)$. After applying customer, provider and service rules, the applicable mappings from the predefined set $M$ are $\left(f c_{1},\left\{s_{5}, s_{11}\right\}\right) \subseteq m_{1},\left(f c_{3},\left\{s_{7}, s_{12}, s_{20}\right\}\right) \subseteq m_{3},\left(f c_{4},\left\{s_{9}\right.\right.$, $\left.\left.s_{13}\right\}\right) \subseteq m_{4},\left(f c_{13},\left\{s_{9}, s_{13}\right\}\right) \subseteq m_{13} . s_{5}, s_{7}$ and $s_{9}$ are all consultations by REG-GP or FRACGP, and are amalgamated to $s_{9} . s_{11}, s_{12}$ and $s_{13}$ are consultations performed by other medical practitioners, and are amalgamated to $s_{13} . s_{20}$ is a quantitation service. The end result of the amalgamation is that $f c_{1}, f c_{3}, f c_{4}, f c_{13}$ are all amalgamated into $s_{9}$ and $s_{13}$, and $f c_{13}$ to $s_{20}$, resulting in three services overall.

In the service provider pool, $s_{9}$ is offered by $S P_{1}$, $s_{13}$ by $S P_{2}$ and $s_{20}$ is only provided by $S P_{8}$.

This results in two possible service bundles, as depicted in Fig 2. Service bundle 1 is offered by a combination of $S P_{1}$ and $S P_{8}$ and has a total price of $\$ 111.3$. Service bundle 2 is offered by a combination of $S P_{2}$ and $S P_{8}$ and has a total price of $\$ 70.75$. The difference between both bundles is that Service bundle 1 has a higher quality of service as REG-GP and FRAC-GP are general practitioners who have additional qualifications. Depending on the preference and budget of the patient, Service bundle 1 or Service bundle 2 can be chosen.

To avoid over fitting and lower fitting, providing many unnecessary FCs and/or missing important FCs, the framework allows verifying the fitness of the alternative SVNs against the required FCs, which help doctors (and patients) to easily select an SVN.

Although the proposed framework can compose alternative SVNs, it cannot apply the so-called ServiceRules yet, i.e. the application of such rules is still a human-based task. Once an SVN is selected by the medical doctor, $\mathrm{s} /$ he has to amalgamate the services in case it is needed. Nonetheless, the given SVN provides the starting point for a doctor to give the final recommendation in terms of services. 


\section{Discussion and future work}

Type 2 Diabetes management focuses on a number of biological parameters related to hypertension (blood pressure control), hyperlipidaemia (lipid control) and hyperglycaemia (blood glucose control). We currently only use blood pressure and blood glucose data in our AAL framework. Lipid control can currently not be performed in the context of remote healthcare using wireless sensors. For this reason the rules (and corollary treatment recommendations and functional consequences) pertaining solely to lipid control have not been executed in the calculations of FCs for patients in the current framework. However, the rules are present and can be used by the framework when desired, because lipid control is an essential element of diabetes management. This points to some gaps indicating future directions.

The relevance of information in patient profiles is currently dictated by its use in the context of the framework, i.e. information that is not used is not relevant. In the case of lipids, we would need to add another cycle in the SVN composition to increase the validity of the framework. This cycle would suggest (until technology advances: non-sensor-based) lipids measurement in certain situations before suggesting other services, thus offering the full gamut of measurements in the context of diabetes. The lipid measurements would need to be recorded in a way that can be understood by the rule execution program.

This opens up the discussion of the importance of patient profiles. We currently focus on diabetes management, but various kinds of information are relevant to different healthcare service domains. For example in palliative care, an acuteness dimension would be added to the framework: people need to be helped quickly in emergency situations (for example after falling on the ground). Patient location thus becomes an important - perhaps in some cases overruling - factor.

Another important aspect of patient profiles is budget: we currently offer different cost options without having a good idea of what the patient can afford. In Australia, the future model of home care will be one where patients are capped on a yearly basis [21]. Cost becomes an important factor in such a model, and patient budgets become an area of optimisation of SVN composition. The aggregated individual SVN optimisations lead to optimisations on a national health policy level.

We would also like to extend our framework to include services performed by friends and relatives, for example in the case of palliative care.

Another important aspect to address in the future is to formally include the Critique, Modify, and Verify
SVN subtasks in the framework. Where the individual outcomes have been verified for this paper, we would need a pilot environment consisting of more medical health practitioners to perform the subtasks in a realworld setting (resulting in actual services being delivered to the patients).

Furthermore, we would like to eventually address all AAL challenges presented in [2], with the subsequent focus being on AAL Challenge 1: 'Adaptivity' and AAL Challenge 8: 'Integration'.

Finally, we will extend the framework to be able to handle ServiceRules in an automated manner, rather than the current manual approach.

\section{Conclusion}

We have demonstrated that our approach solves some of the technical challenges of the AAL domain and contributes to the field of SVN composition.

By integrating the 'medical practice' side of healthcare with the 'business' side of it through the application of a service value network approach we offer solutions to AAL Challenge 7: 'Integration'.

By applying a service value network approach using existing medical data we essentially open up the playing field for service providers and offer solutions to problems pertaining to AAL Challenge 3: 'Heterogeneity'.

By extracting and formalizing rules and characteristics from both the NHMRC guidelines and MBS listings, we offer solutions to the problems posed by AAL Challenge 4: 'Formalization'.

Furthermore, we have extended the field of SVN composition by replacing current assumptions that customers are actively involved in explicating their service requirements with a more tacit approach where requirements are derived from patterns in sensor readings (and corollary diagnosis) based on validated rules on the customer side as well as the supplier side.

With the degree of automation, formalization and integration currently provided by the framework, we could argue that we are well on our way to create a form of 'sensor-driven, automated healthcare'.

\section{Acknowledgements}

We would like to acknowledge the invaluable contributions of Prof. Moyez Jiwa by validating and reviewing many aspects of this paper and the framework in his role of medical practitioner and healthcare domain expert. 


\section{References}

1. Ambient Assisted Living Joint Programme, Catalogue of Projects (2011), Available from: http://www.aaleurope.eu/projects/AALCatalogueV3.pdf, Accessed on 26 March 2012.

2. Kleinberger T., Becker M., Ras E., Holzinger A., and Müller P., "Ambient Intelligence in Assisted Living: Enable Elderly People to Handle Future Interfaces", In: Stephanidis, C.(ed.) UAHCI2007 (Part II). LNCS, vol. 4555, Springer, Heidelberg, 2007, pp 103-112,

3. Eurostat Press Office, "Population Projections 20082060", Available from: http://europa.eu/rapid/pressReleasesAction.do?reference $=$ STAT/08/119, Accessed on 26 March 2012.

4. Australian Bureau of Statistics, ABS Australian Social Trends 4201.0. ABS, Canberra, 2009.

5. Australian Institute of Health and Welfare, "Impact of rurality on health status", Available from: http://www.aihw.gov.au/rural-health-impact-of-rurality/, Accessed on 8 June 2012.

6. Turner, K.J., Docherty, L.S., Wang, F. and Campbell, G.A. "Managing home care networks," in ICN'09, R. Bestak, L. George, V. S. Zaborovsky, and C. Dini, Eds. Los Alamitos, USA: IEEE Computer Society, Mar 2009, pp. 354-359.

7. National Health and Medical Research Council. NHMRC's role. Available from: $<$ http://www.nhmrc.gov.au/about/organisationoverview/nhmrcs-role $>$ Accessed on 4 June 2012

8. Department of Health and Ageing. Medicare Benefits Schedule Book. Australian Government, Canberra (2011)

9. Razo-Zapata, I., De Leenheer, P., Gordijn, J., Akkermans, H., "Service Network Approaches", In Barros, A., Oberle, D. (eds.): Handbook of Service Description: USDL and its Methods, Springer, 2011, pp. 45-74.

10. Hamilton, J., "Service value networks: Value, performance and strategy for the services industry",
Journal of Systems Science and Systems Engineering, vol. 13(4), 2004, pp. 469-489.

11. Allee, V, "A value network approach for modeling and measuring intangibles", In: Transparent Enterprise Conference, 2002.

12. Lovelock, C.H.; Wirtz, J., Services Marketing: People, Technology, Strategy - 7th Edition, Pearson Higher Education, 2010.

13. Basole, R.C., Rouse, W.B., "Complexity of service value networks: Conceptualization and empirical investigation", IBM Systems J. 47(1), 2008.

14. Gordijn, J. and Akkermans, H., "Value-based Requirements Engineering: Exploring Innovative eCommerce Ideas", Requirements Engineering Journal, Vol. 16(4), 2003, pp. 114-134.

15. Akkermans, J.M., et al.: Value Webs: Using Ontologies to Bundle Real-World Services, IEEE Intelligent Systems 19 (4), 2004, pp. 57-66.

16. Gordijn, J. De Leenheer, P. and Razo-Zapata, I. Generating service valuewebs by hierarchical configuration: An ipr case. In Proceedings of HICSS 44, 2011.

17. Razo-Zapata, I., Gordijn, J., De Leenheer, P. and Akkermans, H.. Dynamic cluster-based service bundling: A value-oriented framework. In IEEE 13th Conference on Commerce and Enterprise Computing, 2011.

18. Razo-Zapata, I., De Leenheer, P., Gordijn, J., and Akkermans, H. Service value networks for competencydriven educational services: A case study. In 6th international BUSITAL workshop, 2011.

19. Razo-Zapata, I., De Leenheer, P., Gordijn, J., Akkermans, H. Fuzzy verification of service value networks. CAISE, CAiSE Workshops, 2011, pp. 81-92.

20. Meersman, D.; Debruyne, C.: Purchase Intent, Online Offers and Product Innovation: Misunderstandings in the Ménage à Trois. BIS Workshops 2011, 2011, pp. 132-143.

21. Gillard, J., and Butler, M. More Choice, Easier Access and Better Care for Older Australians (Press release). Australian Government, Canberra, 2012. 